\title{
Desenvolvimento de função de transferência para previsão de área trincada em pavimentos asfálticos por meio da simulação do dano por fadiga utilizando modelo S-VECD e análises elásticas
}

\author{
Development of transfer function for crack area prediction in asphaltic pavements by
} fatigue damage simulation using S-VECD model and elastic analysis

\author{
Lucimar da Silva Santiago¹, Lucas Feitosa de A. L. Babadopulos², Jorge Barbosa Soares ${ }^{3}$ \\ 1Universidade Federal do Cariri, Ceará - Brasil, lucimar.santiago@ufca.edu.br \\ 2Universidade Federal do Ceará, Ceará - Brasil, babadopulos@ufc.br \\ 3Universidade Federal do Ceará, Ceará - Brasil, jsoares@det.ufc.br
}

\section{Recebido: \\ 1 de dezembro de 2018 \\ Aceito para publicação: \\ 9 de março de 2019 \\ Publicado: \\ 31 de agosto de 2020 \\ Editor de área: \\ Francisco Thiago Aragão}

\section{Palavras-chaves:}

Dimensionamento.

Pavimentos asfálticos.

Fadiga.

Dano.

Percentual de área trincada.

Keywords:

Design.

Asphaltic pavements.

Fatigue.

Damage.

Percentage cracked area.

DOI:10.14295/transportes.v28i3.1900

\section{RESUMO}

A evolução dos métodos de caracterização e da quantidade de materiais testados em âmbito nacional nas últimas décadas possibilitou a organização de um banco de dados de pavimentos rodoviários. São informações essenciais para métodos de dimensionamento, cujas funções de transferência possibilitam a previsão do desempenho a partir de modelagem computacional. O principal objetivo do presente trabalho é desenvolver uma função de transferência que associa dados de caracterização de dano por fadiga em laboratório à área trincada observada em campo. As ferramentas usadas foram a rigidez da mistura, caracterizada pelo módulo complexo, e a vida de fadiga, pelas curvas de integridade ( $C$ vs $\mathrm{S}$ ) e de envoltória de falha (GR vs Nf), associados à análise elástica multicamadas e a uma variável de dano "reduzida". Os resultados de evolução de dano calculada foram usados para prever observações da evolução do percentual de área trincada em campo. Numa etapa de calibração envolvendo 27 trechos experimentais, foi possível se obter uma função de transferência única entre as previsões de laboratório e as observações de campo. A validação da função foi realizada com dados de 17 outros trechos. Através de 2 exemplos de dimensionamento, observou-se uma questão importante relativa à definição convencional da variável de dano médio reduzido, sobretudo na previsão de comportamento de pavimentos com espessura de revestimento maior que $15 \mathrm{~cm}$.

\section{ABSTRACT}

The evolution of materials characterization methods and of the amount of materials tested nationwide in the past decades made it possible to organize a road pavements database. These are essential information for pavement design with transfer functions that allow performance prediction from computational modeling. The main objective of this work is to develop a transfer function associating laboratory fatigue damage characterization data to surface cracked area observed in field. The tools used were the asphalt stiffness, characterized by the complex modulus, and the fatigue life, characterized by the integrity curve ( $\mathrm{C}$ vs $\mathrm{S}$ ) and the failure envelope (GR vs Nf), and associated with multilayer elastic analysis and a reduced damage variable. Calculated damage evolution results were used to predict field observations of percent cracked areas. In a calibration phase using 27 test sections, it was possible to obtain a unique transfer function between laboratory predictions (in terms of calculated damage) and field observations (in Terms of percent cracked area). The validation of the transfer function was performed with 17 other sections. Through 2 pavement design examples, it was observed an important aspect of the conventional definition of the average reduced damage variable, especially in the prediction of pavements with surface course greater then $15 \mathrm{~cm}$. 


\section{INTRODUÇÃO}

O método de dimensionamento de pavimentos asfálticos amplamente utilizado no Brasil e ainda em vigor pelo Departamento Nacional de Infraestrutura de Transportes (DNIT) tem mais de meio século. Apenas recentemente apontou-se com a implantação de um novo método, de cunho mecanístico-empírico, a despeito do longo histórico de trabalhos desenvolvidos na academia nacional (Motta, 1991; Benevides, 2000; Franco, 2007). Tanto os trabalhos acadêmicos nacionais quanto o novo método de dimensionamento passaram pelo projeto Rede de Tecnologia em Asfalto (RTA), coordenado pela Petrobras. 0 referido projeto contou com a participação do DNIT e de diversas universidades responsáveis pela coleta de dados e ações técnico-científicas. Um resultado significativo da RTA foi a construção de um banco de dados de materiais utilizados na pavimentação asfáltica nacional, sua caracterização laboratorial e a observação e documentação de seu correspondente comportamento em campo, em proporções não antes existente no país. Sublinha-se, mesmo quando comparado a outros conjuntos de dados coletados internacionalmente, a relevância deste esforço na organização de observações de campo e de laboratório, dispostas num banco de dados, o Sistema da Rede de Tecnologia em Asfalto (SRTA). Essas informações armazenadas permitem fazer, por meio da utilização de metodologias coerentes, e para diferentes proposições de sistemas de dimensionamento, correspondências entre resultados de ensaios laboratoriais e de desempenho em vias reais.

Os pavimentos asfálticos podem apresentar diversos tipos de defeitos, sendo os considerados mais impactantes nas vias brasileiras a deformação permanente e o trincamento por fadiga. Sobre a deformação permanente, no Brasil, destacam-se os trabalhos de Guimarães (2009), cujo foco é os materiais granulares, e os de Nascimento (2008), Batista et al. (2017) e Almeida Jr et al. (2018), que se concentraram no estudo da deformação permanente em misturas asfálticas. Quanto à fadiga, existem diversos trabalhos científicos que investigam a deterioração de pavimentos no Brasil, no que toca ao relacionamento entre dados de laboratório e o desempenho de pavimentos em campo, destacando-se as contribuições de Nascimento (2015a) e Fritzen (2016). Esse é também o objetivo do presente trabalho, ou seja, apresentar um framework de previsão de área trincada ao longo da vida útil do pavimento utilizando o dano por fadiga calculado como dado de entrada, cujo procedimento de obtenção pode ser encontrado em Santiago et al. (2018).

Os dados laboratoriais e de desempenho em campo de trechos monitorados no âmbito da RTA foram utilizados como suporte ao desenvolvimento do presente trabalho. As simulações foram realizadas num programa de dimensionamento de pavimentos asfálticos, o CAP3D-D, um desdobramento do programa de análise estrutural CAP3D (Holanda et al., 2006) que inclui a previsão de dano e de área trincada, entre outros melhoramentos.

\section{FUNDAMENTAÇÃO TEÓRICA}

\subsection{Precedentes}

O dano por fadiga em misturas asfálticas pode ser entendido como uma perda progressiva de resistência do material asfáltico quando submetido a um número de repetições de carga. No caso de esse número ser igual ou superior à capacidade do material em resistir a essas repetições, deve ocorrer falha (caso do pavimento em campo) ou ruptura por fadiga (caso do ensaio em laboratório). Esse tipo de dano está diretamente associado ao fenômeno de trincamento em campo (Nascimento, 2015a; Fritzen, 2016). Assim como nos referidos trabalhos, a chamada "função de transferência" (equação que traduz resultados de laboratório em uma previsão de 
desempenho em campo, no caso, de um percentual de área trincada), cujo desenvolvimento é apresentado em seções subsequentes, parte da utilização de resultados simulados computacionalmente de dano por fadiga. Um passo a passo de como obter os resultados de dano em misturas asfálticas para previsão de falha em campo, a partir de uma análise elástico-linear e à luz da metodologia Simplified Viscoelastic Continuum Damage (S-VECD), utilizado no presente trabalho é apresentado em Santiago et al. (2018). A metodologia se inspira nos trabalhos de Nascimento (2015a) e em desenvolvimentos do grupo da North Carolina State University (NCSU). No que diz respeito a caracterização das misturas asfálticas, baseia-se na Teoria do Potencial de Trabalho de Schapery e de seu princípio da correspondência elástico-viscoelástico, que utiliza a definição de pseudo-deformações (Schapery, 1984; Park et al., 1996), para propor uma metodologia de análise simplificada de dano em revestimentos asfálticos. Note-se que os modelos do CAP3D-D objetivam uma análise simplificada com relação ao usado pelo grupo da NCSU. 0 programa utiliza uma abordagem elástica linear para o cálculo estrutural de tensões e deformações, a qual exige esforço computacional bem inferior à abordagem viscoelástica. Uma simulação viscoelástica ocorre ao longo do tempo, cada passo de tempo no cálculo requerendo esforço equivalente ao de uma única análise elástica. Apesar de elástica linear a uma dada temperatura e frequência, a análise incorporada ao CAP3D-D possibilita a inserção de parâmetros próprios de viscoelasticidade, tais como a dependência do comportamento do pavimento quanto à temperatura e velocidade de passagem de veículos, além da distribuição horária do tráfego. Trata-se de uma análise elástica linear correspondente à análise viscoelástica linear em caso de carregamento senoidal, algo que deriva do Princípio da Correspondência Elástico-Viscoelástica (Biot, 1955). Considera-se como dados de entrada tanto os dados de módulo dinâmico da mistura asfáltica (curvas mestras), quanto dados do ensaio de fadiga por tração-compressão direta (obtidos por meio da AASHTO TP 107). Após a realização das análises no CAP3D-D, é fornecido um relatório no qual, para cada mês do período de projeto, é apresentado um resultado de "dano médio", obtido para o número de ciclos correspondente. Entende-se por dano médio o somatório do "dano" calculado em cada ponto de análise, abaixo da carga, devidamente dividido pelo número total de pontos (Santiago et al., 2018). 0 dano no CAP3D-D é definido como o consumo relativo de vida de fadiga (número de ciclos aplicados com a deformação do ponto analisado, dividido pelo número máximo de ciclos que pode ser aplicado no material nessa mesma deformação, sem que haja ruptura por fadiga). As principais características dos três trabalhos de referência do presente artigo são expostas na Tabela 1.

Tabela 1 - Características gerais dos trabalhos de Nascimento (2015a), Fritzen (2016) e Santiago et al. (2018).

\begin{tabular}{llll}
\hline Parâmetro & Nascimento (2015a) & Fritzen (2016) & Santiago et al. (2018) \\
\hline Análise & Viscoelástica & Elástica linear & Elástica linear \\
\hline Rigidez do revestimento & $\begin{array}{l}\text { Módulo de relaxação obtido a } \\
\text { partir do módulo dinâmico }\end{array}$ & Módulo de resiliência & Módulo dinâmico \\
\hline Fadiga & Tração-compressão direta & Compressão diametral & Tração-compressão direta \\
\hline $\begin{array}{l}\text { Área de cálculo do dano } \\
\text { médio }\end{array}$ & 110 pontos abaixo da carga & 110 pontos abaixo da carga & $\begin{array}{l}\text { 240 pontos de Gauss (120 abaixo da } \\
\text { carga e o restante na sua zona de } \\
\text { influência) }\end{array}$ \\
\hline Temperatura & Considerada na análise & Não considerada & Considerada na análise \\
\hline $\begin{array}{l}\text { Distribuição horária e } \\
\text { anual do tráfego }\end{array}$ & Considerada na análise & Não considerada & Considerada na análise \\
\hline
\end{tabular}




\subsection{Trincamento em misturas asfálticas}

O fenômeno de trincamento em campo é traduzido, na pavimentação, como um percentual de área trincada (\%AT) do pavimento. Esse índice leva em consideração a extensão total do trecho analisado e a área julgada por técnicos como trincada. Para determinação da área trincada, pode-se fazer uso de técnicas como o processamento digital de imagem (Scheffy et al., 1999) e sistemas de fotogrametria de pavimentos (Kertész et al., 2008), ou, de maneira mais simples, com uso de grades metálicas (LAPAV, 2011) ou acompanhamento visual (RTA, 2010). Nesta última, utilizada para obtenção dos dados adotados neste trabalho, a extensão da via que se deseja avaliar é dividida em trechos de $20 \mathrm{~m}$ de comprimento, os quais são subdivididos virtualmente em 10 partes longitudinais e em 3 partes transversais, resultando em 30 células virtuais de análise de defeitos (de $2 \mathrm{~m} \times 1 / 3$ da largura da via). Essa divisão é feita para facilitar a coleta de defeitos, uma vez que a inspeção é visual e realizada por dois técnicos que percorrem a extensão do trecho analisado. Os técnicos devem identificar a existência de trinca em uma célula. Caso isso ocorra, considera-se que ela está $100 \%$ trincada. Se essa mesma trinca se estende para outras células, cada célula atingida também deve ser contabilizada como trincada. 0 percentual de AT corresponde à quantidade relativa de células trincadas no total de células avaliadas. Tratase de um procedimento simples, mas que permite uma avaliação razoável do grau de integridade do pavimento quanto ao trincamento por fadiga. Esse foi o método escolhido para medição de percentual de AT nos trechos monitorados por Nascimento (2015a) na Ilha do Fundão - RJ, assim como nos demais trechos monitorados no âmbito da Rede Temática de Tecnologia em Asfalto.

Os valores obtidos em campo podem ser plotados em um gráfico percentual de AT x dano (previsto por meio de modelos que envolvem dados de laboratório) correspondente à data de medição em campo. A curva resultante pode ser representada por uma sigmoidal, como considerado no MEPDG (Mechanistic Empirical Pavement Design Guide) norte americano (Equação 1), ou por um modelo de potência, como o proposto por Nascimento (2015a) (Equação 2). A curva de Nascimento (2015a) foi a que melhor se relacionou aos dados brasileiros.

onde: $\quad$ AT: $\quad$ percentual de área trincada que se origina no topo do revestimento e desce até sua base;

$\mathrm{C}_{1}$ e $\mathrm{C}_{2}$ : coeficientes de regressão da curva; $\mathrm{D}$ = dano médio calculado por meio de simulação computacional, correspondente ao mês em que foi realizada a medição de área trincada em campo.

$$
\% A T=C_{1}\left(N / N_{f} \text { red }_{s}\right)^{C 2}
$$

onde: $\quad \mathrm{N} / \mathrm{N}_{\mathrm{f}}$ reds $_{\mathrm{s}}$ : dano médio reduzido;

$\mathrm{C}_{1}$ e $\mathrm{C}_{2}$ : coeficientes de regressão; sendo o Dano médio reduzido = dano médio multiplicado por um fator de deslocamento de dano (que depende da taxa prevista de evolução de dano no pavimento analisado), o que permite correlacionar de maneira mais acurada o dano com o início do trincamento em campo. O leitor é referido a Nascimento (2015a, 2015b) para maiores detalhes sobre o dano reduzido. 
Para obter os resultados de dano por fadiga necessários ao desenvolvimento da "função de transferência", Nascimento (2015a) utilizou o modelo S-VECD aplicado em um modelo de análise estrutural viscoelástico, contido no programa Layered Viscoelastic Continuum Damage (LVECD). Dentre os modelos estatísticos disponíveis, o que melhor se adequou à relação dano por fadiga x percentual de AT previsto (calculado) é representado pela Equação 2.

Fritzen (2016) utilizou como dados de entrada de rigidez o módulo de resiliência, e de fadiga os resultados do ensaio de fadiga por compressão diametral. A análise estrutural foi elástica linear com suporte do programa SisPav Br, uma versão atualizada do programa proposto por Franco (2007). Assim como em Nascimento, o modelo proposto por Fritzen foi o da Equação 2. Nas duas pesquisas também foi determinado um fator de erro da função de transferência, cujo formato segue o modelo da Equação 3. Deve ser observado que o uso dos diferentes coeficientes encontrados com cada um dos sistemas de dimensionamento mencionados não é uma questão de escolha por parte do analista. 0 conjunto de premissas utilizado em cada sistema de dimensionamento está intrinsecamente atrelado às equações obtidas, inclusive de funções de transferência e de erro, assim como seus coeficientes. Dessa maneira, a escolha do sistema de dimensionamento impõe a escolha das equações e coeficientes a serem utilizados.

$$
\text { Erro }=a+\frac{b}{\left(1+e^{c-d * \log \left(100 * N / N_{f} \text { red }_{S}\right)}\right)}
$$

onde: $\quad$ a, b, c, d: coeficientes de ajuste da equação.

\section{METODOLOGIA DE OBTENÇÃO DE FUNÇÃO DE TRANSFERÊNCIA DE PREVISÃO DE PERCENTUAL DE ÁREA TRINCADA E RESULTADOS OBTIDOS}

O desenvolvimento da função de transferência no âmbito da presente pesquisa seguiu em linhas gerais os passos metodológicos propostos por Nascimento (2015a). A principal diferença é a forma de obtenção do dano por fadiga previsto no pavimento, apresentado em Santiago et al. (2018) e que foi resumido nos itens 2.1 e 3.2 .

\subsection{Breve caracterização dos trechos}

Os trechos experimentais cujos dados foram usados nesta pesquisa são divididos em dois tipos: i) 27 construídos na Ilha do Fundão - RJ, monitorados pela COPPE/UFRJ e Cenpes/Petrobras. São nomeados de TE (trecho experimental): 1, 4, 7, 11, 19, 34, 37, 40, 43, 45, 46, 47, 48, 50, 55, $58,60,63,65,67,70,73,75,86,88,89$ e 93; e,

ii) 17 trechos monitorados por outras universidades participantes do projeto: 2 da USP, 2 da UFSC, 1 da UFRGS, 2 da UFSM, além de 10 da Rodovia Nova Dutra.

As propriedades das misturas desses trechos (e.g. valores dos parâmetros da curva sigmoidal do módulo dinâmico da mistura asfáltica e de fadiga por tração-compressão direta) e os dados de percentual de AT observada em campo, respectivamente utilizados para a simulação de dano e posterior correlação com o trincamento por fadiga medida em campo, constam em Nascimento (2015a). Destaca-se que os dados dos 27 trechos da Ilha do Fundão foram usados para calibração de uma função de transferência, obtida neste trabalho para os modelos de comportamento material e de estrutura de pavimento adotados. Dos 44 trechos mencionados nesta pesquisa, esses são os que apresentam maior quantidade de levantamentos de percentual de AT em campo, existindo pelo menos 4 pontos observados em campo para cada trecho. Como visto adiante, isso permite montar curvas da relação percentual de AT observada x Dano que 
melhor representam a realidade, sendo assim, mais adequadas à calibração de uma função de transferência. Já as temperaturas consideradas para as análises de dano, em cada um dos trechos, foram retiradas das Normais Climatológicas do Brasil 1961-1990, publicadas pelo Instituto Nacional de Meteorologia (INMET) (2017). Foram escolhidos os dados de temperatura coletados nas estações meteorológicas mais próximas dos trechos, conforme indicado na Tabela 2.

Tabela 2 - Estações meteorológicas indicativas das temperaturas dos trechos considerados.

\begin{tabular}{ll}
\hline Estação & Trechos/Quantidade \\
\hline São Bento (Duque de Caxias - RJ) & Ilha do Fundão/27 \\
Taubaté - SP & Nova Dutra/10 \\
Franca - SP & USP/2 \\
Araranguá - SC & UFSC/2 \\
Porto Alegre - RS & UFRGS/1 \\
Santa Maria - RS & UFSM/2 \\
\hline
\end{tabular}

As temperaturas mínimas foram consideradas para o horário de análise de 00:00 às 08:00h, as máximas para o horário de 08:00 às 16:00h e as médias para o horário de 16:00 às 24:00h. De posse dessas informações e do tráfego nos trechos, que também consta no trabalho de Nascimento (2015a), prossegue-se para a determinação do dano médio.

\subsection{Obtenção do dano médio}

Para obtenção do dano médio em cada mês do período de projeto de cada trecho monitorado, faz-se uso do procedimento descrito em Santiago et al. (2018). Em resumo, tem-se como input: o tráfego inicial do trecho, o seu percentual anual de crescimento, a sua distribuição anual, a sua distribuição horária diária, a estrutura do pavimento (espessuras das camadas), o módulo de resiliência das camadas granulares e coeficientes de Poisson, os dados da curva sigmoidal representando o módulo dinâmico da mistura asfáltica e os dados da curva de fadiga obtida por meio do ensaio de fadiga por tração-compressão direta.

Após a inserção dos dados, inicia-se a análise, a qual, no CAP3D-D, para exemplos semelhantes aos deste artigo, despende de 3 a $10 \mathrm{~min}$, a depender da capacidade do processador do computador. Ao final obtém-se, um relatório (em formato .txt) no qual, para cada mês, estão associados os valores de $\mathrm{N}$ mensal, de $\mathrm{N}$ acumulado e o dano médio. No caso das análises desta pesquisa, o período de projeto estimado foi de 180 meses. Os resultados estão resumidos na Figura 1.

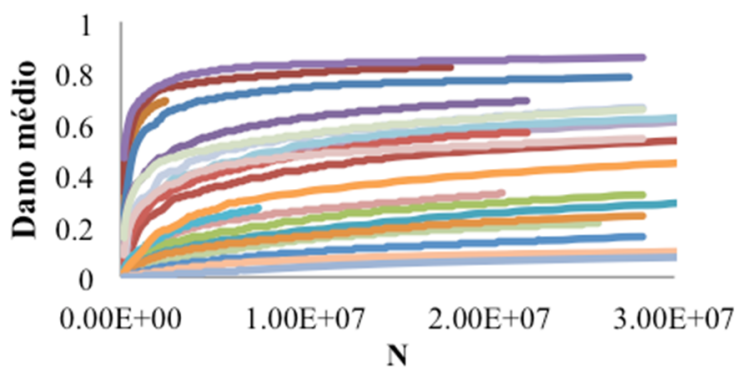

Figura 1. Gráfico dos danos médios em função do $\mathrm{N}$, obtidos nas análises realizadas nesta pesquisa para diferentes trechos 


\subsection{Determinação do dano médio correspondente ao percentual de AT observado e o percentual de AT correspondente ao crescimento inicial do dano médio}

Conforme apresentado no item 2.2, o percentual de AT observado em campo advém de acompanhamento visual. Buscam-se então, no relatório de dano médio, os valores de dano médio correspondentes às datas de coleta do percentual de AT em campo. São construídos gráficos individuais para cada trecho do percentual de AT x dano médio correspondente. A Figura 2 apresenta o conjunto das informações desses trechos, sendo possível observar que aproximadamente a partir dos $10 \%$ de AT é que os dados de percentual de AT tendem a crescer de maneira mais acentuada com o aumento do dano. Por isso, o dano médio a $10 \%$ de AT será o valor usado para correlacionar com uma taxa inicial de crescimento de dano, conforme explicado a seguir. Esse valor de dano é obtido por regressão nos gráficos individuais de cada trecho.

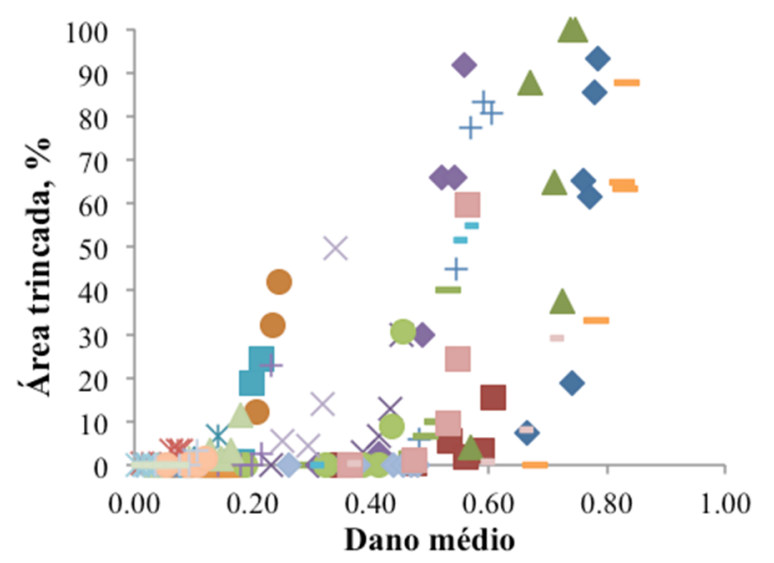

Figura 2. Gráfico de percentual de AT observado em campo x dano médio correspondente

\subsection{Dano inicial correspondente a $10 \%$ AT}

Da Figura 1 é possível perceber que, no início da vida útil do pavimento, as curvas de dano são bem mais inclinadas, ou seja, nesse trecho do gráfico o dano aumenta mais rapidamente com o tempo. Chama-se esse fator de crescimento observado inicialmente de taxa inicial de crescimento de dano. Assim, baseado nos gráficos como os da Figura 1, escolhe-se visualmente um valor de crescimento de dano que possa melhor representar o conjunto de dados. No caso da pesquisa ora apresentada, esse conjunto de dados é formado por 27 curvas, representativas dos 27 trechos experimentais considerados. Destaca-se que se diferentes conjuntos de hipóteses e modelos utilizados para as análises estruturais tivessem sido usados, isso levaria a formatos de curvas de evolução diferentes, mesmo partindo de um mesmo conjunto de dados. Isso seria compatibilizado com diferentes funções de transferência relacionando a previsão de laboratório e a observação de campo. Em Nascimento (2015a) o valor obtido de taxa inicial de crescimento de dado foi de 0,35 . Neste estudo, embora outros valores tenham sido testados, também o valor de 0,35 foi considerado o mais adequado para a calibração de dados experimentais de laboratório e de campo. As razões para esta escolha são apresentadas no item 3.7.

\subsection{Determinação do mês correspondente ao crescimento inicial de dano}

De posse do valor do crescimento inicial do dano determinado para o conjunto de dados da análise, coletam-se, nos relatórios de dano, os meses em que ocorre o dano médio de 0,35 (T0,35). 
Com essas informações constrói-se o gráfico de dano a 10\% AT x T0,35, apresentado na Figura 3. Através dele, é possível verificar uma alta correlação entre os valores de dano a $10 \%$ AT e o tempo necessário para ocorrer o crescimento inicial de dano considerado $(0,35$, neste estudo).

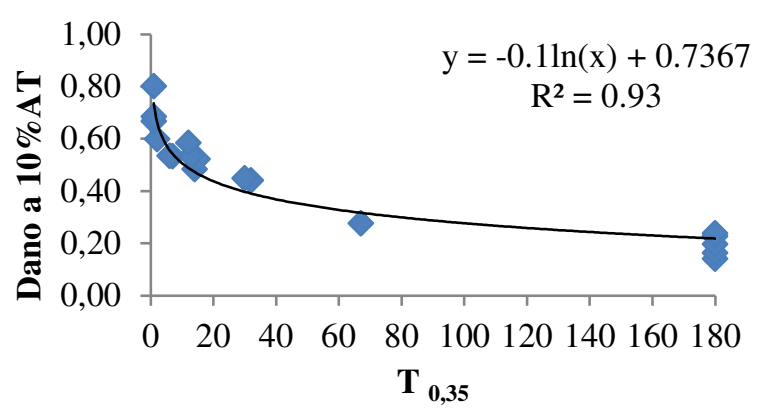

Figura 3. Gráfico de $10 \%$ AT $\times \mathrm{T}_{0,35}$

\subsection{Obtenção dos danos médios reduzidos e da função de transferência}

No gráfico da Figura 2, percebe-se uma dispersão entre os pontos do gráfico para diferentes trechos observados, o que dificultaria estabelecer uma correlação adequada entre os resultados obtidos para fins de obtenção de curvas únicas úteis para o dimensionamento. Para resolver essa questão, Nascimento (2015a) sugeriu utilizar um valor de ajuste (translação) horizontal que aproximasse os pontos das diferentes curvas de evolução de trincamento. A variável no eixo horizontal obtida após esse ajuste é o dano reduzido. 0 dano reduzido pode ser obtido por meio de uma das Equações 4 e 5 (duas opções diferentes de regra para translação).

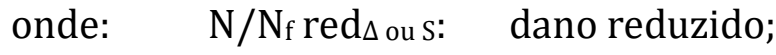

$$
\begin{aligned}
& N / N_{f} \operatorname{red}_{\Delta}=N / N_{f}+\Delta \\
& N / N_{f} \operatorname{red}_{S}=N / N_{f} \times S
\end{aligned}
$$

$\mathrm{N} / \mathrm{N}_{\mathrm{f}}$ : dano médio;

$\Delta$ ou S: $\quad$ shift factor.

Para encontrar as equações de $\Delta$ e S, é necessário estabelecer um valor central de dano reduzido, na direção do qual cada uma das curvas de evolução de trincamento será transladada. Nascimento (2015a) utilizou o valor de 0,5 , contudo neste estudo o valor que mais se alinhou com os dados foi de 0,4. As Figuras $4 \mathrm{a}$ e $4 \mathrm{~b}$ apresentam os gráficos com as melhores correlações obtidas com $\Delta$ (Equação 4) e $S$ (Equação 5) com o $\mathrm{T}_{0,35}$, respectivamente.

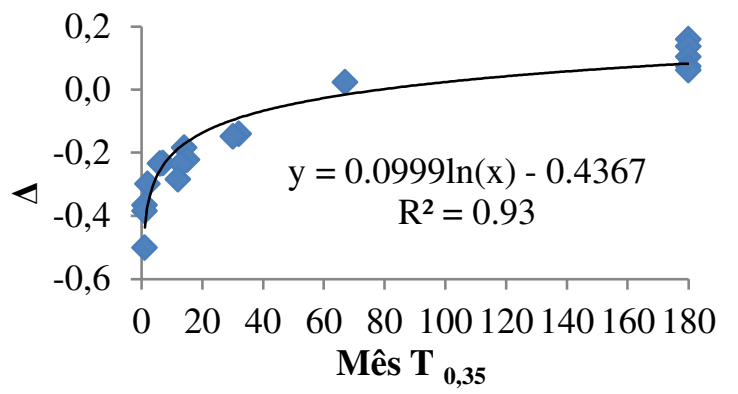

(a)

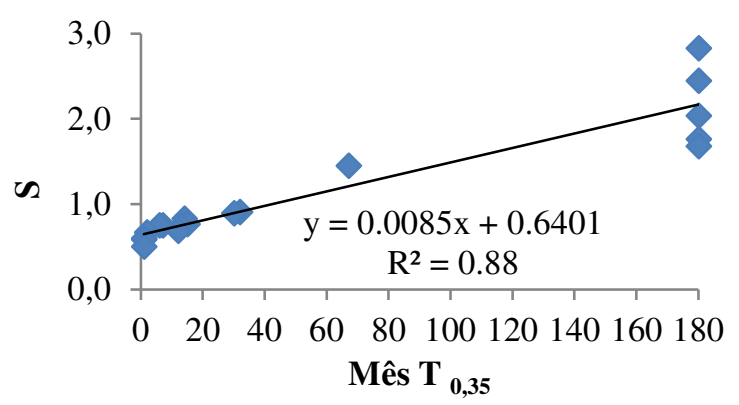

(b)

Figura 4. Correlação entre (a) $\Delta$ e $\mathrm{T}_{0,35}$ e (b) $\mathrm{S}$ e $\mathrm{T}_{0,35}$ 
Apesar de a melhor correlação obtida neste estágio da investigação ter sido aquela com a função $\Delta$ (Figura 4a), os dados de percentual de AT previsto tiveram uma melhor aderência à previsão de área trincada com a utilização da função $S$ (Figura 4b), de maneira que apenas esta segunda foi utilizada nos passos a seguir. Além disso, preferiu-se a utilização da tendência linear com a função S. A Figura 5 mostra a curva de percentual de AT em função do dano reduzido obtido usando a Equação 5.

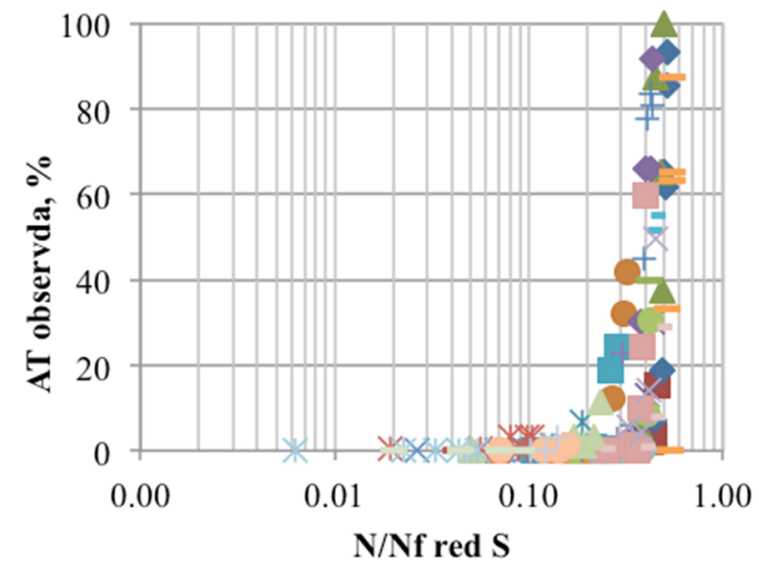

Figura 5. Curva única obtida para a evolução do trincamento nos trechos experimentais

Observa-se que o dano médio reduzido (valores no eixo x) não chega a 0,60. Deve-se frisar que cada ponto no revestimento tem o consumo de vida de fadiga (dano tal qual definido neste artigo) limitado a 1. A partir de um certo tempo de simulação, alguns dos pontos que participam da média para o cálculo do dano (240 pontos de Gauss, sendo 120 abaixo da carga e o restante na sua zona de influência, conforme Tabela 1 e detalhes em Santiago et al., 2018) chegam ao valor limite de 1. Esses pontos não danificam mais segundo o modelo, que limita o dano a 1. Enquanto isso, os demais pontos, por estarem submetidos a deformações menores, se danificam muito lentamente. Dessa maneira, o comportamento observado é, na verdade, esperado. É importante sublinhar que decisões diferentes de valor central de dano reduzido na direção do qual cada uma das curvas de evolução de trincamento seriam transladadas interfeririam no valor observado de 0,60 de dano médio reduzido máximo. A tendência de comportamento dos trechos, quanto ao trincamento, por meio das análises aqui realizadas é a de um modelo de potência, tal como em Nascimento (2015a).

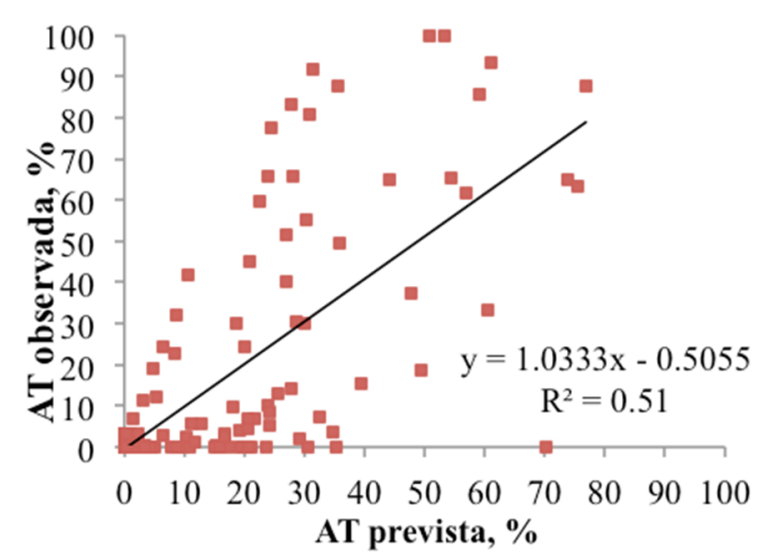

Figura 6. Correlação entre o percentual de AT observado e o percentual de AT previsto 
A correlação entre o percentual de AT observado em campo e o percentual de AT previsto a partir de informações do pavimento e de dados de laboratório apresenta um valor de 51\%, como indica a Figura 6. Em outros métodos de dimensionamento presentes na literatura, como o MEPDG norte-americano, este valor é de 27\%. Ainda assim, sublinha-se que a importância neste ponto é a incorporação de mecanismos de falha e de considerações sobre o comportamento do material mais realistas e o delineamento claro do processo de modelagem do comportamento da estrutura e de sua falha.

Os coeficientes da função de potência que representa o conjunto de dados obtido (curva de percentual de AT $\mathrm{x}$ dano médio reduzido) foram os escolhidos para compor a função de transferência de percentual de AT, objeto deste trabalho. As Equações 6 e 7 apresentam a função shift fator (SF) e a função de transferência (útil para o dimensionamento, para obter o percentual de AT com uma curva única a partir de dados de laboratório), respectivamente, ambas ajustadas após a utilização da ferramenta Solver do Excel, que teve como objetivo reduzir o erro geral entre os valores globais do percentual de AT observado em campo e o percentual de AT previsto através da função de transferência.

$$
S F=0,00002\left(T_{0,35}\right)^{2}+0,00872\left(T_{0,35}\right)+0,65294
$$

onde: $\quad \mathrm{T}_{0,35}$ : $\quad$ em meses em que o dano atinge o valor de 0,35 para cada trecho analisado, cujo maior valor admissível é 67.

$$
\% A T=721,96589\left(\mathrm{~N} / \mathrm{N}_{f} \text { red }_{s}\right)^{3,76808}
$$

onde: $\quad \mathrm{N} / \mathrm{N}_{\mathrm{f}}$ reds: dano médio reduzido calculado para o mês analisado.

\subsection{Análise paramétrica para determinação do dano inicial}

No item 3.4, citou-se que o valor do crescimento inicial do dano foi de 0,35 . A fim de determinar o melhor valor dessa taxa de crescimento, para o conjunto de dados analisados, foram realizadas calibrações da função de transferência considerando taxas iniciais de 0,25, 0,35 e 0,45. 0 objetivo era verificar qual desses valores apresentava resultados com melhor correlação entre o percentual de AT observado e o percentual de AT previsto. Já de início, a análise com o T0,45 apresentou baixa correlação com uma curva de potência que, como determinado por Nascimento (2015a), teria melhor aderência com os resultados dos trechos analisados. Para as outras duas taxas, verificou-se dois fatores: i) o menor valor do somatório das diferenças quadradas entre o percentual de AT observado e o percentual de AT previsto (após ajuste minimizando quadrados); e, ii) a correlação entre o conjunto de dados gerados entre o percentual de AT observado e o percentual de AT previsto, como apresentado na Figura 6. Os maiores valores de $\mathrm{R}^{2}$ $(0,51$, cf. Figura 6$)$ foram obtidos com as análises feitas com a taxa inicial de crescimento de 0,35 .

\subsection{Validação da função de transferência de percentual de AT obtida}

Um passo importante no processo de obtenção de uma função de transferência e após o processo de calibração (objeto dos itens 3.1 a 3.6) é a validação da função obtida. Para isso, necessita-se de dados de laboratório e de campo, oriundos de trechos experimentais diferentes daqueles utilizados na etapa de calibração. Na presente pesquisa, utilizaram-se os dados de um total de 17 trechos nacionais da RTA, conforme descrito no item 3.1. 
Os passos para validação são os seguintes:

- determina-se o dano médio (calculado) dos trechos experimentais, considerando uma análise de 180 meses;

- de posse desses resultados, utiliza-se a equação percentual de AT calibrada, para obtenção dos percentuais de AT previstos dos trechos experimentais da validação;

- com esses resultados é possível verificar a acurácia e a exatidão da função de transferência gerada através da aderência entre os resultados de percentual de AT observado e o percentual de AT previsto da etapa de calibração (pontos vermelhos) e da etapa de validação (pontos azuis), tal como pode ser visto na Figura 7;

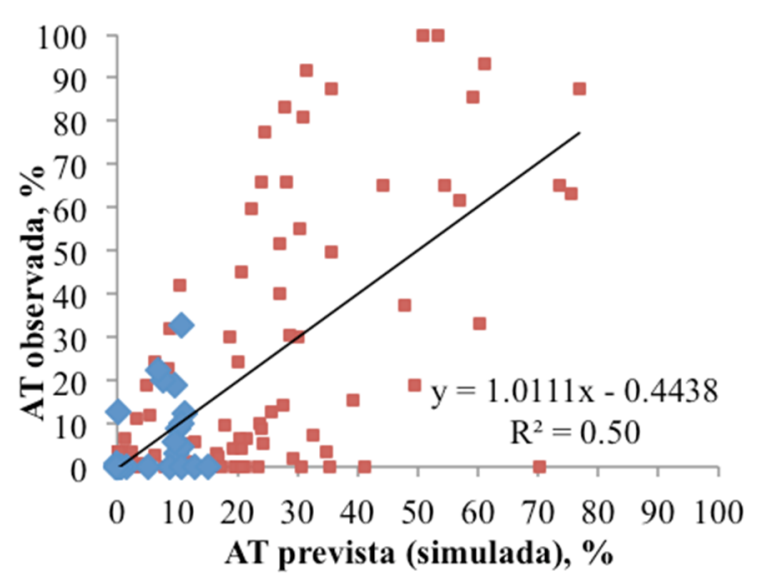

Figura 7. Correlação entre o percentual de AT observado e o percentual de AT previsto

- foi gerada, então, a função de erro que pode ser aplicada a cada trecho. Diferentemente do MEPDG norte-americano e de Nascimento (2015a), a função erro (erro-padrão, EP) obtida neste trabalho tem um formato de curva exponencial (Equação 8).

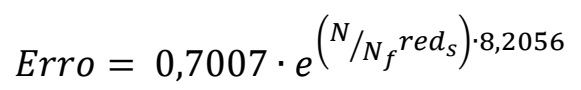

- por fim, essa equação permite acrescentar ao dimensionamento proposto no âmbito desta pesquisa o conceito de confiabilidade. Assim, para previsão de percentual de AT com determinado nível de confiança, deve-se utilizar a Equação 9.

$$
\% A T=721,96589\left(N / N_{f} r e d_{s}\right)^{3,76808}+0,7007 \cdot e^{\left(N_{N} / N_{f} \text { red }_{s}\right) \cdot 8,2056} \cdot Z_{p}
$$

onde: $\quad Z_{p} \quad$ desvio normal padrão que varia conforme o nível de confiabilidade estabelecido para cada análise (por exemplo, para $50 \%=0$, para $99 \%=2,33$ ).

\subsection{Dimensionamento teste}

Neste subitem é apresentado um dimensionamento de pavimento utilizando o procedimento de previsão de percentual de AT proposto nesta pesquisa. Será usado o conceito de intervalo de confiança para os cálculos de previsão. Foram escolhidas duas misturas asfálticas: UFSC 50/70 e UFSM 50/70. A Figura 8 apresenta a estrutura do pavimento teste, que, com exceção das espessuras da camada asfáltica, será a mesma em todas as análises. As espessuras da camada asfáltica testada são: $5 \mathrm{~cm}, 10 \mathrm{~cm}, 15 \mathrm{~cm}$ e $20 \mathrm{~cm}$. Assume-se um tráfego inicial anual de 5,0E+06 e uma taxa de crescimento anual de 3\%. Deve ser observado que, para fins didáticos, as camadas granulares foram consideradas como elásticas lineares, mas que o programa CAP3D-D permite 
a simulação considerando modelos resilientes. A camada asfáltica tem, em cada ponto, módulo de elasticidade igual ao módulo dinâmico da mistura na temperatura e na frequência correspondente à velocidade de passagem dos veículos, ou seja, $\mathrm{E}=\left|\mathrm{E}^{*}\right|(\mathrm{T}, f)$.

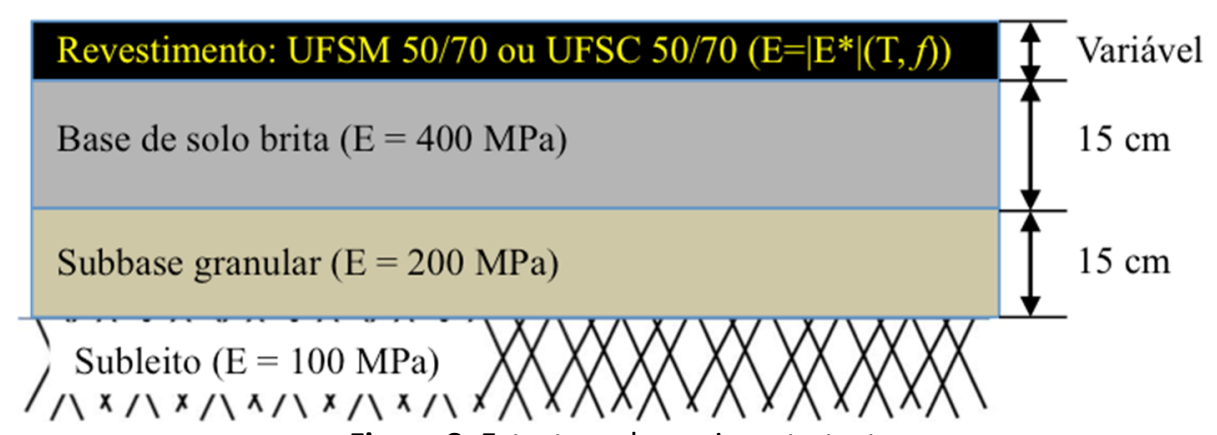

Figura 8. Estrutura do pavimento teste

Os danos médios são calculados para cada espessura e cada mistura asfáltica proposta (8 análises de dano ao todo). Em seguida, utiliza-se a função de transferência para determinar o percentual de AT previsto para cada situação, em duas condições de confiabilidade: $50 \%$ $(Z p=0,00)$ e 99\% (Zp=2,33). Esses resultados são então plotados em gráficos do percentual de AT previsto em função de 120 meses (horizonte de projeto comum no Brasil). Os resultados obtidos são apresentados na Figura 9.

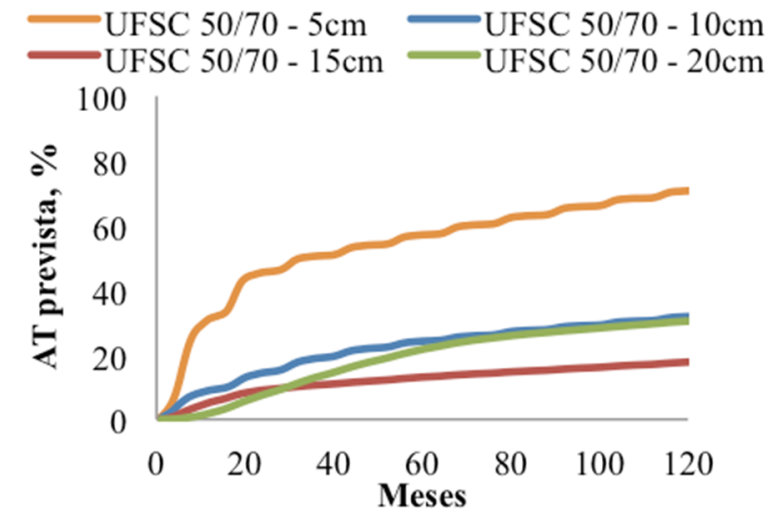

(a)

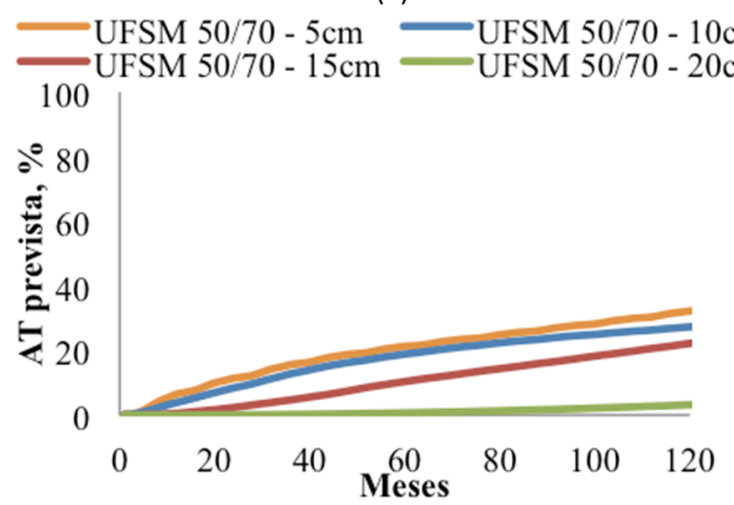

(c)

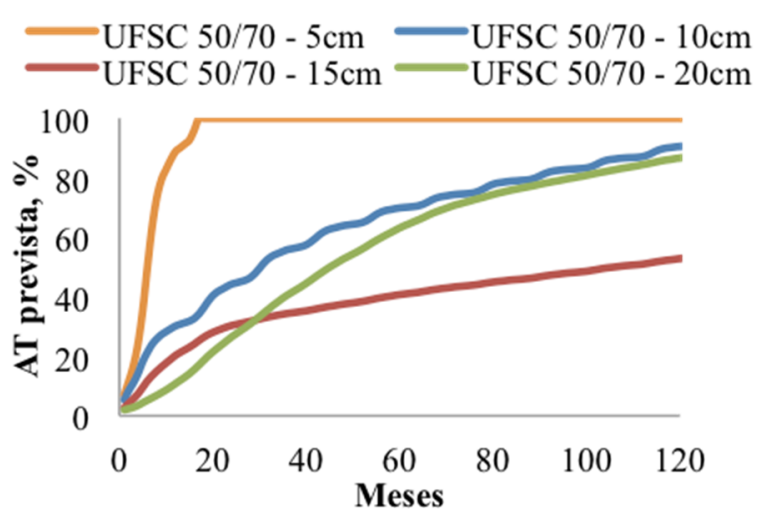

(b)

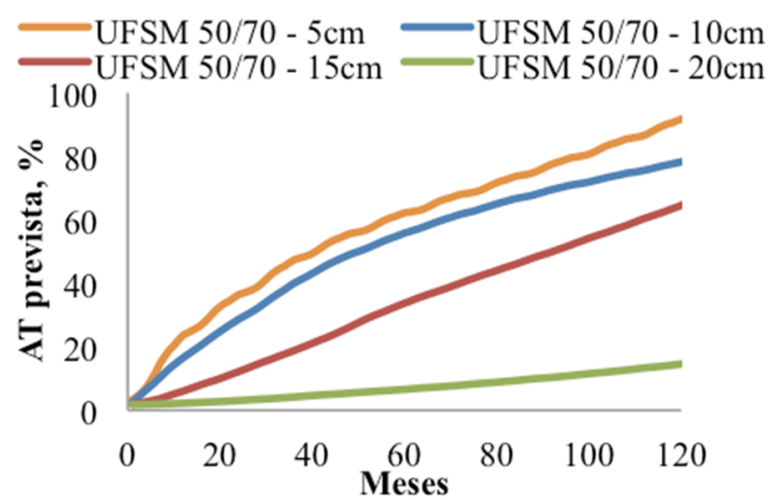

(d)

Figura 9. Evolução prevista da área trincada em função do tempo com diferentes espessuras para (a) Mistura UFSC 50/70 com 50\% de confiabilidade; (b) Mistura UFSC 50/70 com 99\% de confiabilidade; (c) Mistura UFSM 50/70 com 50\% de confiabilidade; (d) Mistura UFSM 50/70 com 99\% de confiabilidade 
Considerando os primeiros 30 meses, para as 4 situações apresentadas, o crescimento de percentual de AT foi condizente com a tendência esperada: revestimentos mais esbeltos apresentam trincamento mais rapidamente do que revestimentos mais espessos. No entanto, no caso da estrutura da UFSC, esse sentido se inverte a partir dos 30 meses, para a espessura de $20 \mathrm{~cm}$. Buscou-se, então, verificar o que estaria provocando essa distorção, no que se refere ao dano. 0 gráfico da Figura 10a mostra que o dano médio calculado está condizente com o esperado: revestimentos mais esbeltos apresentam danos médios maiores que aqueles mais espessos. Notou-se, no entanto, que o mesmo não ocorreu em relação ao dano reduzido. Na Figura $10 \mathrm{~b}$, nota-se uma quase coincidência entres os resultados de $10 \mathrm{~cm}$ e $20 \mathrm{~cm}$ a partir de cerca de 40 meses, o que não é condizente com o esperado.

A passagem matemática entre o dano médio e o dano médio reduzido acarreta esse comportamento das curvas de evolução de percentual de AT previsto. A fim de resolver tal questão foram realizadas novas análises na fase de calibração, contudo a tendência dos resultados foi a mesma para determinadas misturas e estruturas. Deve-se observar, porém, que, se as curvas forem analisadas apenas até os valores típicos de análise de área trincada para fins de projeto (em torno de 20\%), não há inconsistências, de maneira que não é invalidado o uso da previsão de comportamento para áreas trincadas em torno de $20 \%$. Isso fica evidenciado na Figura 11 , que contém os mesmos dados que a Figura 9b, mas com as escalas ajustadas para mostrar o comportamento até $30 \%$ de área trincada.

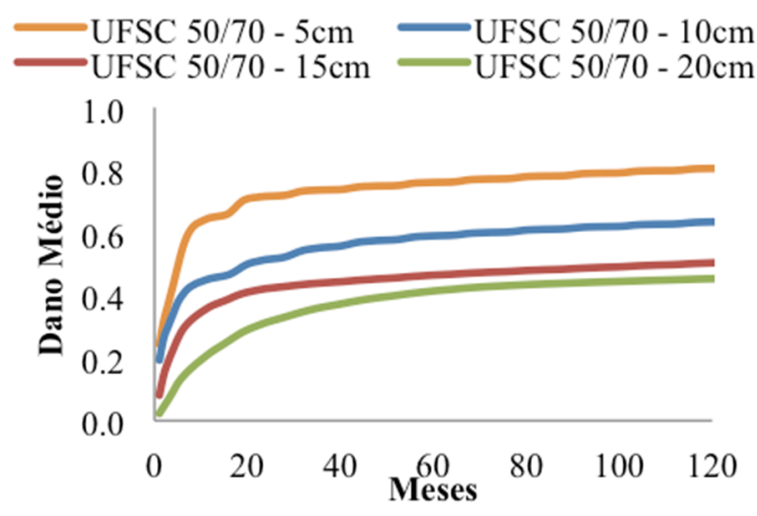

(a)

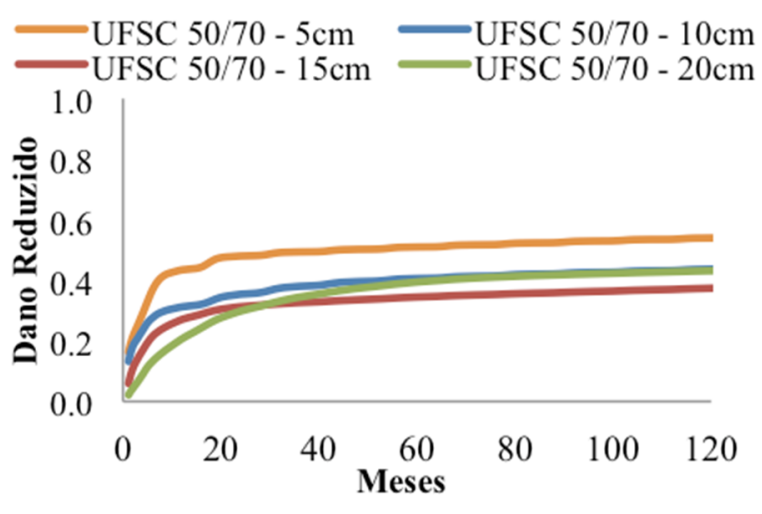

(b)

Figura 10. (a) Dano médio em função do tempo e (b) dano reduzido em função do tempo para mistura UFSC

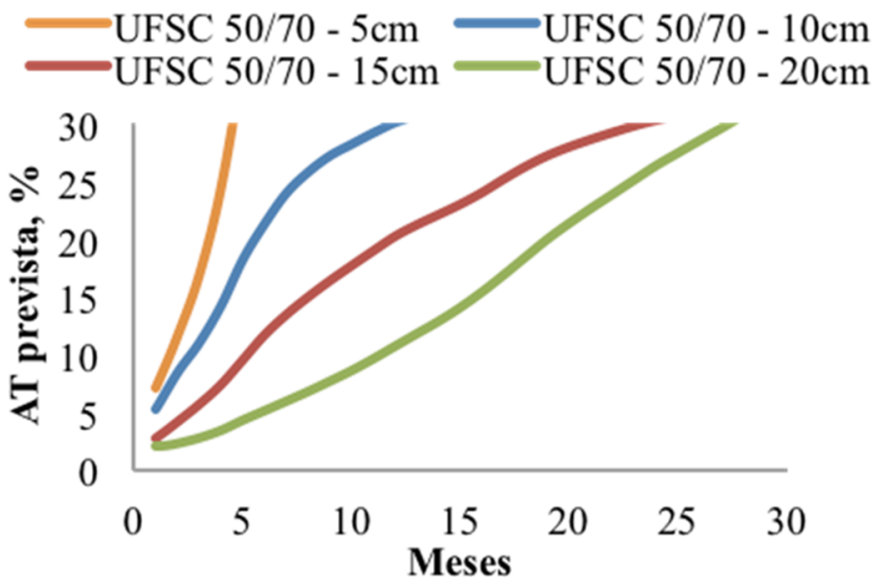

Figura 11. Mistura UFSC 50/70 com 99\% de confiabilidade (mesmos dados da Figura 10b), com escala apresentando dados até $30 \%$ de área trincada 
Apesar da distorção relatada, percebe-se que, para as estruturas com revestimento de até $15 \mathrm{~cm}$ (o que ocorreu para ambas as misturas e pavimentos estudados), a previsão do percentual de AT ao longo do tempo está coerente com o comportamento esperado. Trata-se, portanto, das espessuras mais comuns dos pavimentos nacionais. Além disso, apesar de a distorção poder ser observada na curva de evolução de área trincada quando extrapolada até níveis muito severos de dano, para níveis adotados na prática (de cerca de 20 ou 30\%), a distorção não ocorre. Por outro lado, conforme observado na Figura 12, percebe-se que maiores valores de confiabilidade levam a se prever menor tempo para os mesmos valores de AT, i.e., a análise é mais conservadora. No caso da mistura UFSC $50 / 70 \mathrm{com} 15 \mathrm{~cm}$ de espessura, para $50 \%$ de confiabilidade, a análise prevê que a estrutura somente chegará a 30\% AT após 180 meses. Já para 99\% de confiabilidade, este valor é previsto com 24 meses de vida de serviço. Neste sentido, os valores de confiabilidade a serem aplicados no dimensionamento devem ser criteriosamente pensados pelo analista, devendo ser levada em consideração a importância da via e os recursos disponíveis para sua concepção.

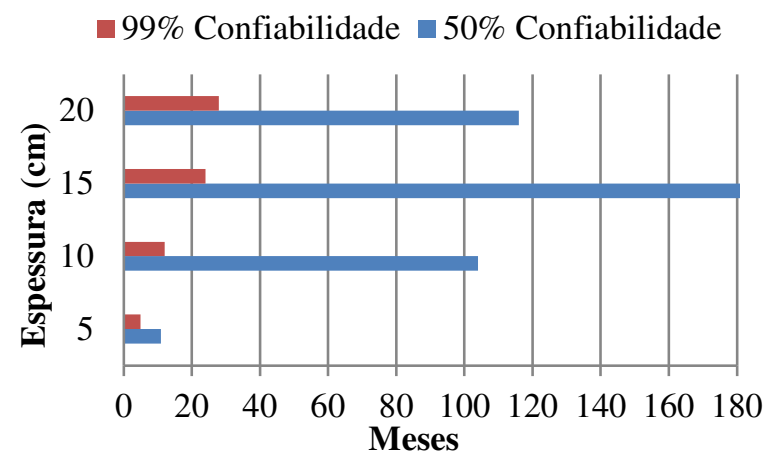

(a)

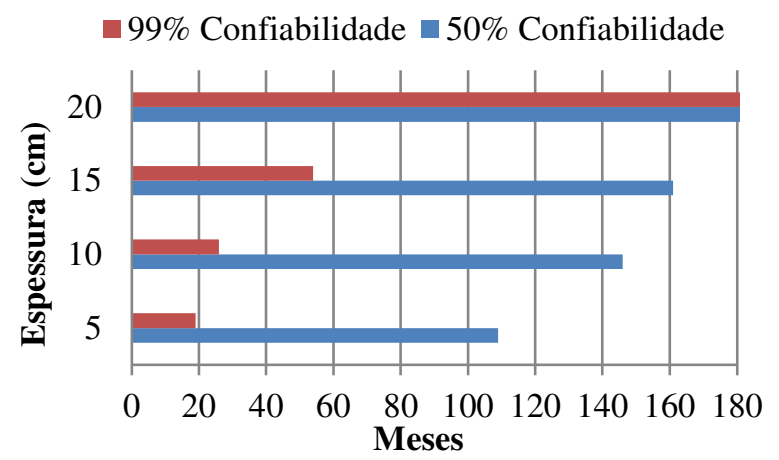

(b)

Figura 12. Tempo em que as misturas (a) UFSC 50/70 e (b) UFSM 50/70 alcançam 30\% de AT para análises com 50\% e com $99 \%$ de confiabilidade

\section{CONCLUSÕES E RECOMENDAÇÕES}

Foi executado com sucesso o processo de calibração e validação de uma função de transferência para o CAP3D-D com as hipóteses de cálculo ora utilizadas no software. Isso levou a um sistema de dimensionamento capaz de levar em consideração o fenômeno de fadiga ocorrendo com diferentes temperaturas e velocidades de passagem de veículos. Essa consideração é particular a modelos de material que incluem viscoelasticidade, adequados à previsão de comportamento de misturas asfálticas. 0 sistema apresentado encontra-se em estágio suficientemente desenvolvido para uso intuitivo por profissionais ligados ao projeto de pavimentos, com interface amigável. Ele foi usado ainda para um exercício de dimensionamento que atesta o bom funcionamento para diferentes pavimentos, com diferentes materiais, dentro dos limites esperados para aplicações de dimensionamento.

Foi observado para pavimentos com espessura acima de $15 \mathrm{~cm}$ e com notada severidade de previsão de trincamento (mais de 30\%) que pode ocorrer uma inconsistência de previsão (pavimentos mais robustos que deterioram mais rapidamente) devido ao modo como é calculado o dano médio reduzido. Sublinha-se porém, que este efeito é observado apenas para estágios de evolução muito severa de área trincada (acima de 30\%), que não interferem em projetos que considerem áreas trincadas inferiores, por exemplo 20\%, como tipicamente adotado. É importante destacar que o fenômeno de trincamento de pavimentos em campo é bastante complexo 
e pode envolver questões como o trincamento por cisalhamento, considerado mais comum em revestimentos com espessuras maiores.

Por fim, acredita-se que a particularização dos bancos de dados para a elaboração de funções de transferência de área trincada regionais possibilitará melhoria da previsão de área trincada. Isso devido ao fato de que os materiais, as condições climáticas e de tráfego numa perspectiva mais local (estadual ou regional) se aproximarão mais da realidade do que tratar localidades tão distintas sob uma perspectiva nacional, ainda mais num país com as dimensões do Brasil. Sugere-se, portanto, que sejam fortalecidas ações de pesquisa estaduais ou regionais para a construção de funções de transferência a partir de bancos de dados locais e suficientemente robustos.

\section{REFERÊNCIAS}

AASHTO TP 107 (2014) Determining the damage characteristic curve of asphalt concrete from direct tension cyclic fatigue tests. American Association of State Highway and Transportation Officials, Provisional Standard TP 107, Washington, DC.

ALMEIDA JR, P. O.; F. D. BOEIRA; L. P. SPECHT; T. C. CERVO; D. S. PEREIRA; R. CENTOFANTE; V. S. BARBOZA JR; C. F. C. SILVA (2018) Avaliação laboratorial do tipo e teor de ligante e da granulometria na deformação permanente de misturas asfálticas. Revista Transportes. Rio de Janeiro. v. 26, n. 2, p. 1-15. DOI: 10.14295/transportes.v26i2.1407.

BATISTA, J. B. S.; L. F. A. L. BABADOPULOS; J. B. SOARES (2017) Relationship between multiple stress creep recovery (MSCR) binder test results and asphalt concrete rutting resistance in Brazilian roadways. Construction and Building Materials, v. 145, p. 20-27. DOI: 10.1016/j.conbuildmat.2017.03.216.

BENEVIDES, S.A.S. (2000) Análise Comparativa dos Métodos de Dimensionamento de Pavimentos Asfálticos: Empírico do DNER e da Resiliência da COPPE/UFRJ em Rodovias do Estado do Ceará. 2000. Dissertação de Mestrado. Universidade Federal do Rio de Janeiro. Rio de Janeiro.

BIOT, M.A. (1955) Dynamics of Viscoelastic Anisotropic Media. In: Midwestern Conference on Solid Mechanics. Engineering, 4. , 2006, Lafayette, USA. Proceedings [...]. Lafayette: Experiment Station. v. 129.

FRANCO, F.A.C.P. (2007) Método de Dimensionamento Mecanístico-Empírico de Pavimentos Asfálticos - SISPAV. Tese de Doutorado. Universidade Federal do Rio de Janeiro. Rio de Janeiro.

FRITZEN, M.A. (2016) Desenvolvimento e Validação de Função de Transferência para Previsão de Dano por Fadiga em Pavimentos Asfálticos. Tese de Doutorado. Universidade Federal do Rio de Janeiro. Rio de Janeiro.

HOLANDA, A.S.; E. PARENTE Jr.; T. D. P. ARAÚJO; L. T. B. MELO; F. EVANGELISTA Jr.; J. B. SOARES, (2006). Finite Element Modeling of Flexible Pavements. In: Iberian Latin-American Congress on Computational Methods in Engineering (CILAMCE), 27, 2006, Belém, Pará. Proceedings [...]. Belém, Pará. p. 1-14.

INMET (2017) Normais Climatológicas do Brasil 1961-1990. Instituto Nacional de Meteorologia. Ministério da Agricultura Pecuária e Abastecimento. Brasília, DF. Disponível em: <http://www.inmet.gov.br/portal/index.php?r=clima/normaisClimatologicas>. Acesso em: 19 jun. 2017.

KERTÉSZ, I.; T. LOVAS; A. BARSI, (2008) Photogrammetric pavement detection system. In: INTERNATIONAL SOCIETY FOR PHOTOGRAMMETRY AND REMOTE SENSING, 21., 2008, Beijing, China. Proceedings [...]. Beijing, China: IAPRS. v. 27, n. B5, p. 897-902. Disponível em: <https://www.isprs.org/proceedings/XXXVII/congress/5_pdf/156.pdf>. Acesso em: 04 mar. 2017.

LAPAV (2011) PROJETO DE PESQUISA CONCEPA - LAPAV “Estudo de Desempenho de Pavimento Experimental com objetivo de validar método racional de dimensionamento de Pavimentos flexíveis”. Laboratório de Pavimentação Escola de Engenharia - UFRGS. Porto Alegre, RS.

MOTTA, L.M.G. (1991) Método de Dimensionamento de Pavimentos Flexíveis; Critério de Confiabilidade e Ensaios de Cargas Repetidas. Tese de Doutorado. Universidade Federal do Rio de Janeiro, Rio de Janeiro.

NASCIMENTO, L.A.H. (2008) Nova Abordagem da Dosagem de Misturas Asfálticas Densas com Uso do Compactador Giratório e Foco na Deformação Permanente. Dissertação de Mestrado. Universidade Federal do Rio de Janeiro. Rio de Janeiro.

NASCIMENTO, L.A.H. (2015a) Implementation and Validation of the Viscoelastic Continuum Damage Theory for Asphalt Mixture and Pavement Analysis in Brazil. Tese de Doutorado. North Carolina State University. Raleigh-USA.

NASCIMENTO, L.A.H. (2015b) Implementação e Validação da Teoria do Dano Contínuo Viscoelástico para a Análise de Misturas e Pavimentos Asfálticos no Brasil. Asfalto em Revista. Rio de Janeiro. n. 42 . p. 1 -25.

PARK, S. W.; KIM, Y. R.; SCHAPERY, R. A. (1996) Viscoelastic continuum damage model and its application to uniaxial behavior of asphalt concrete. Mechanics of Materials. v. 24, n. 4, p. 241-255. DOI: 10.1016/S0167-6636(96)00042-7.

RTA (2010) Manual de Execução de Trechos Monitorados. Rede Temática de Tecnologia em Asfalto. Rio de Janeiro: Petrobras SA.

SANTIAGO, L.S.; TORQUATO E SILVA, S.A.; SOARES, J.B. (2018) Determinação do dano em pavimentos asfálticos por meio da combinação do modelo S-VECD com análises elásticas. Revista Transportes. Rio de Janeiro. v. 26, n. 2, p. 31-43. DOI: 10.14295/transportes.v26i2.1446. 
SCHAPERY, R.A. (1984). Correspondence Principles and a Generalized J-integral for Large Deformation and Fracture Analysis of Viscoelastic Media. International Journal of Fracture, v. 25, p. 195-223. DOI: 10.1007/BF01140837.

SCHEFFY, C.; COETZEE, N.; DIAZ, E. (1999) Asphalt Concrete Fatigue Crack Monitoring and Analysis Using Digital Image Analysis Techniques. In: Accelerated Pavement Testing 1999 International Conference. Reno-USA. 1., 1999, Reno, USA. Proceedings [...]. Reno, EUA: APT. p. 1-21. Disponível em: <http://citeseerx.ist.psu.edu/viewdoc/download?doi=10.1.1.61.4478\&rep=rep1\&type=pdf>. Acesso em: 04 mar. 2017. 